\title{
Les collisions d'oiseaux à l'édifice Marly à Sainte-Foy, Québec, de 1978 à 2007
}

\author{
YVAN POULIOT \\ 239 Marie-Victorin, Saint-Nicolas, Québec, G7A 2W9 Canada; courriel: ypyp@sympatico.ca
}

Pouliot, Yvan. 2008. Les collisions d'oiseaux à l'édifice Marly à Sainte-Foy, Québec, de 1978 à 2007. Canadian Field-Naturalist 122(2): 153-157.

Since its construction in 1978, thousands of birds have been killed following collisions on the Marly mirror-glassed building located in the western area of Quebec City. This paper presents ornithological observations and describes the collision phenomenon at this site. Data show that the number of birds killed by collisions has declined dramatically over the 30-year period. Probable causes of this decline are discussed.

Key Words: bird-window collisions, fatalities, building, decline, population, habitat, Quebec.

Mot-clés: oiseaux, collisions, édifice, vitre-miroir, mortalité, population, déclin, habitat, Québec.

Les collisions d'oiseaux contre des constructions humaines causent chaque année la mort de millions d'oiseaux en Amérique du Nord. Certains spécialistes estiment ce nombre à près d'un milliard (Klem 1990; Dunn 1993; Doeker 2005*). Ces collisions s'expliquent de différentes façons:

(A) Structures éclairées la nuit (édifices, tours de télécommunication, ponts, etc.): les oiseaux sont attirés et éblouis par la lumière;

(B) Structures hautes: placées sur leur route, les oiseaux s'y frappent par temps de mauvaise visibilité;

(C) Verre transparent: les oiseaux ne voient pas les parois vitrées et s'y heurtent;

(D) Verre réfléchissant: l'environnement se réfléchit dans la vitre. Lorsque la vitre est de type miroir, l'effet est parfait. L'oiseau vole allègrement vers l'image de son environnement et se cogne contre la vitre;

(E) L'oiseau voit son image dans une vitre miroir. S'il est un mâle en période de reproduction, il fonce illico sur l'intrus "mâle virtuel" pour défendre son territoire et frappe le verre.

Au printemps 1978, l'auteur travaille sur le chantier de construction de l'édifice Marly de Sainte-Foy située à $4 \mathrm{~km}$ à l'ouest du pont de Québec. Chaque jour avant et après le travail, les oiseaux qui se sont tués en se frappant contre les vitres miroir de l'édifice sont ramassés. Le nombre et les espèces d'oiseaux recueillis à cette époque, ainsi qu'au cours d'autres visites effectuées par la suite sur les lieux, sont résumés dans cet article.

\section{Aire d'étude et Méthodologie}

Dans les années 1970, l'ensemble du secteur était occupé par une forêt naturelle composée de plusieurs associations matures, notamment une érablière à érable à sucre (Acer saccharum), une chênaie rouge (Quercus ruber), une frênaie (Fraxinus spp.) et une hêtraie (Fagus gradifolia). Situé dans un corridor mig- ratoire (fleuve Saint-Laurent/rivière Cap-Rouge), ce milieu forestier dénommé boisé Marly, regorgeait d'oiseaux, en particulier à l'automne et au printemps (Reginald Ouellet, communication personnelle: Notes de rencontres du 23 août 2000 et du 17 novembre 2006). Cette forêt, d'une superficie d'environ 250 hectares à l'époque, est maintenant fragmentée par l'urbanisation.

La construction de l'édifice Marly débute en 1976 et se termine en 1979. L'édifice est entièrement recouvert de vitres miroir dorées (pellicule d'or) dans son pourtour, créant une surface réfléchissante d'environ 14000 mètres carrés. Le boisé se trouve à plus de 100 mètres de l'édifice à l'exception du côté nord-est où il se trouve à moins de 10 mètres.

L'étude repose sur une série d'observations ponctuelles effectuées au cours de visites des lieux au printemps et à l'automne entre 1978 et 2007. Ces visites, au nombre de 148 , consistaient à marcher le long des façades de l'édifice, à ramasser les oiseaux trouvés morts et à les placer dans des sacs pour être identifiés le soir même. Les visitent comportaient des périodes d'observations d'une durée variant entre 5 et 95 minutes, pour un total de 3045 minutes. Les informations suivantes étaient consignées: nombre, espèce, sexe (lorsque le dimorphisme le permettait), durée d'observation, date et endroit de cueillette (orientation de la façade).

\section{Résultats}

Dans l'ensemble, un total de 181 oiseaux représentant 38 espèces ont été trouvés et répertoriés (voir annexe). L'importance relative des différents groupes d'oiseaux les plus affectés par les collisions est présentée au Tableau 1.

Les collisions observées contre l'édifice Marly correspondent majoritairement à la situation " $\mathrm{D}$ " décrite en introduction. Des cas de comportement d'attaque 
TABLEAU 1. Oiseaux victimes de collision à l'édifice Marly, 1978-2007 ( $n=181)$

\begin{tabular}{|c|c|c|c|}
\hline Groupe d'espèces & Printemps & Automne & Total \\
\hline Parulines (warblers) & $63,6 \%$ & $26,1 \%$ & $39,8 \%$ \\
\hline Juncos (juncos) & $1,5 \%$ & $22,6 \%$ & $14,9 \%$ \\
\hline Gros-becs et cardinals (grosbeaks \& cardinals) & $6,1 \%$ & $17,4 \%$ & $13,3 \%$ \\
\hline Grives (thrushes) & $21,2 \%$ & $6,9 \%$ & $12,2 \%$ \\
\hline Roitelets (kinglets) & $0 \%$ & $13,0 \%$ & $8,3 \%$ \\
\hline Bruants (buntings) & $1,5 \%$ & $8,7 \%$ & $6,1 \%$ \\
\hline Pics et grimpereaux (woodpeckers \& creepers) & $1,5 \%$ & $2,6 \%$ & $2,2 \%$ \\
\hline Mésanges (chickadees) & $0 \%$ & $2,6 \%$ & $1,6 \%$ \\
\hline Quiscales (grackles) & $3,0 \%$ & $0 \%$ & $1,1 \%$ \\
\hline Viréos (vireos) & $1,5 \%$ & $0 \%$ & $0,5 \%$ \\
\hline
\end{tabular}

d'un mâle contre son image miroir (situation "E") ont également été observés à quelques reprises. Les oiseaux qui se heurtent ne constituent pas nécessairement un portrait représentatif de l'avifaune du boisé, mais reflètent d'abord les espèces qui ont une propension à se cogner dans les vitres pour des raisons comportementales. Le secteur abrite plusieurs espèces qui ne se cognent jamais ou très rarement, notamment celles qui suivent qui ont été observées de temps à autres dans le boisé:

- Colibri à gorge rubis (Archilochus colubris)

- Corneille d'amérique (Corvus brachyrhynchos)

- Etourneau sansonnet (Sturnus vulgaris)

- Geai bleu (Cyanocitta cristata)

- Hirondelle bicolore (Tachycineta bicolor)

- Jaseur d'amérique (Bombycilla cedrorum)

- Merle d'amérique (Turdus magratorius)

- Mésange à tête noire (Poecile atricapillus)

- Moineau domestique (Passer domesticus)

- Pic flambloyant (Caolaptes auratus)

- Sittelle à poitrine blanche (Sitta carolinensis)

- Tyran huppé (Myiarchus crinitus)

Les parulines représentent le principal groupe de victimes des collisions $(39,8 \%)$. Elles sont proportionnellement plus affectées au printemps $(63,6 \%)$ qu'à l'automne $(26,1 \%)$. La grande majorité des oiseaux (>90\%) se cognent contre la façade située au nordest et ce, autant au printemps qu'à l'automne (données non-présentées). C'est à cet endroit que la forêt se trouve le plus près de l'édifice (environ 10 mètres), et que les arbres et les oiseaux se réflètent pleinement dans les vitres miroir. Les oiseaux qui se cognent semblent être majoritairement des mâles, du moins au printemps ( 23 mâles, 3 femelles et 18 inconnus).

Chacune des espèces observées se trouve dans son aire de nidification. Les oiseaux observés au printemps sont donc des nicheurs potentiels. Certains indices laissent cependant croire qu'il s'agit d'oiseaux de passage puisque la plupart des collisions a lieu durant les périodes de migration.

Le nombre de collisions a diminué radicalement depuis 30 ans. Par exemple, le nombre moyen d'oiseaux trouvés morts par visite est de 2,6 (42 individus, 16 visites) en mai 1978 alors qu'il est de 0,82 (9 indi- vidus, 11 visites) en mai 2000 et de 0,25 ( 1 individu, 4 visites) en mai 2007 (basée sur les données de l'annexe). Il est estimé que le taux de collisions a diminué d'un facteur 10 à 30 depuis les observations de 1978 et de 1980. Cette baisse radicale du taux de collisions suggère un déclin important de la population locale de passereaux. D'autres facteurs que les collisions doivent également être considérés pour expliquer cette baisse, notamment:

- l'effritement de l'habitat forestier dans ce secteur au profit de l'urbanisation;

- l'apprentissage et l'habituation des oiseaux à la présence des vitres miroir;

- un changement du corridor de migration des espèces concernées dans ce secteur;

- l'utilisation de pesticides en milieu urbain et périurbain (la plupart des espèces affectées sont des insectivores);

- une population de prédateurs nécrophages qui s'est probablement développée autour de l'édifice; les oiseaux morts étant ramassés rapidement, ils ne peuvent être répertoriés.

Le phénomène de prédation des oiseaux morts par des nécrophages a été observé indirectement à quelques reprises. Par exemple, des corneilles ont été vues avec des oiseaux dans le bec. Cependant, des observations prolongées (données non présentées) montrent que ce phénomène ne peut à lui seul expliquer la baisse de collisions.

\section{Discussion}

Les données obtenues témoignent de l'abondance (fréquence élevée de collisions) et de la diversité des espèces (50 espèces courantes) qui fréquentaient le boisé il y a plus de 25 ans (voir liste en annexe). On y retrouvait des espèces relativement rares (ex. paruline à ailes dorées), des espèces typiques de forêts matures (ex. paruline à poitrine baie), des espèces de jeunes forêts (ex. paruline à flanc marron), de forêts de conifères (ex. paruline tigrée), de feuillus (ex. grive des bois) ou de milieux semi-ouverts (ex. junco ardoisé). L'absence de données entre 1980 et 2000 ne permet pas de determiner à quel rythme le déclin s'est effectué. Basée sur certaines suppositions, les calculs (non 
présentés ici par manque d'espace) montrent que le nombre d'oiseaux qui ont péri contre les vitres de l'édifice s'élève à plus de 5000 .

La contribution respective des collisions au déclin des espèces étudiées est difficile à établir avec précision. Les données sont toutefois claires sur un aspect: le nombre et la biodiversité des oiseaux fréquentant ce milieu ont chuté significativement depuis 30 ans. Les observations effectuées (non présentées) au cours des dernières années dans le boisé à proximité montrent que celui-ci est occupé aujourd'hui surtout par des espèces ubiquistes ou représentatives de milieux dégradés comme les mésanges, sansonnets, corneilles et moineaux. Le déclin observé ne peut être attribué directement à une tendance générale dans la province ou au Canada car les inventaires d'oiseaux nicheurs (Downes et Collins 2003) montrent que les populations des espèces étudiées dans ce texte n'ont pas diminuées de façon marquée au cours des 3 à 4 dernières décennies.

\section{Conclusion}

Les observations d'oiseaux à l'édifice Marly représentent un exemple tangible de changements notables qui s'opèrent subrepticement dans notre environnement et ce, à l'intérieur de quelques années. Les données de 2006-2007 montrent que malgré un taux de collision relativement très bas, il se tuent encore une centaine d'oiseaux par année. La grande majorité des oiseaux morts depuis 1978 a été retrouvée le long de la façade du côté nord-est, là où des arbres se trouvent situés tous près du mur vitré. Une solution simple au problème de collisions à l'édifice Marly aurait été de couper ces quelques arbres. Il y en aurait moins d'une dizaine à enlever.

\section{Remerciements}

À messieurs Réginald Ouellet, Gervais Lessard et aux réviseurs anonymes pour les commentaires constructifs apportés au manuscrit.

\section{Documents citées}

Decker, R. 2005. Bird Conservation Network Window Glass: the deadliest hazard for birds. www.ben.org.

\section{Littérature citées}

Downes, C. M., et B. T. Collins. 2003. Le relevé des oiseaux nicheurs du Canada de 1967 à 2000. Cahier de biologie, Service canadien de la Faune $\mathrm{N}^{\circ} 219.44$ pages.

Dunn, E. H. 1993. Bird mortality from striking residential windows in winter. Journal of Field Ornithology 64: 302 309.

Klem, D. Jr. 1990. Collisions between birds and windows: mortality and prevention. Journal of Field Ornithology 61: 120-128.

Rosa, Jacques. 1980. Les oiseaux se "cognent" pour mourir. Service canadien de la Faune, Région du Québec, Octobre 1980. $11 \mathrm{p}$

Received 20 November 2007

Accepted 20 February 2009

ANNEXE: Liste des oiseaux trouvés morts au bas des murs vitrés de l'édifice Marly lors de visites effectuées entre 1978 et 2007

\begin{tabular}{|c|c|c|c|c|c|c|}
\hline Année & Date & Espèce & Nom scientifique & Sexe & Nombre & Endroit \\
\hline \multirow[t]{25}{*}{1978} & \multirow[t]{2}{*}{18 mai } & Junco ardoisé & Junco hyemalis & M & 1 & Sud-est \\
\hline & & Paruline couronnée & Seiurus aurocapilla & M & 1 & Nord-est \\
\hline & 21 mai & Grive à dos olive & Catharus ustulatus & & 4 & Nord-est \\
\hline & 22 mai & Paruline obscure* & Vermivora peregrina & M & 1 & Nord-est \\
\hline & 23 mai & Paruline obscure & Vermivora peregrina & M & 2 & Nord-est \\
\hline & \multirow[t]{2}{*}{24 mai } & Paruline obscure & Vermivora peregrina & M & 4 & Nord-est \\
\hline & & Cardinal à poitrine rose & Pheucticus ludovicianus & M & 2 & Sud-est \\
\hline & \multirow[t]{2}{*}{25 mai } & Paruline tigrée & Dendroica tigrina & M & 1 & Nord-est \\
\hline & & Paruline obscure & Vermivora peregrina & M & 5 & Nord-est \\
\hline & \multirow[t]{3}{*}{26 mai } & Paruline obscure & Vermivora peregrina & M & 3 & Nord-est \\
\hline & & Paruline à tête cendrée & Dendroica magnolia & & 1 & Nord-est \\
\hline & & Paruline à calotte noire & Wilsonia pusilla & & 1 & Nord-est \\
\hline & \multirow[t]{7}{*}{29 mai } & Paruline couronnée & Seiurus aurocapilla & M & 1 & Nord-est \\
\hline & & Paruline du Canada & Wilsonia canadensis & $\mathrm{F}$ & 2 & Nord-est \\
\hline & & Paruline obscure & Vermivora peregrina & M & 2 & Nord-est \\
\hline & & Gros-bec errant & Coccothraustes vespertinus & $\mathrm{F}$ & 1 & \\
\hline & & Paruline rayée & Dendroica striata & M & 2 & \\
\hline & & Grive à dos olive & Catharus ustulatus & & 6 & \\
\hline & & $\begin{array}{l}\text { Paruline à poitrine baie } \\
\text { Aucun }\end{array}$ & Dendroica castanea & $\mathrm{F}$ & 2 & \\
\hline & 1 juin & Grive à dos olive & Catharus ustulatus & & 1 & \\
\hline & 2 juin & Aucun & & & & \\
\hline & \multirow[t]{2}{*}{5 juin } & Paruline à gorge orangée & Dendroica fusca & M & 1 & Nord-est \\
\hline & & Grive à dos olive & Catharus ustulata & & 1 & \\
\hline & 6 juin & Aucun & & & & \\
\hline & 7 juin & Paruline triste & Oporornis philadelphia & M & 1 & Nord-est \\
\hline
\end{tabular}


ANNEXE: (suite)

\begin{tabular}{|c|c|c|c|c|c|}
\hline Année & Espèce & Nom scientifique & Sexe & Nombre & Endroit \\
\hline 9 juin & Aucun & & & & \\
\hline 12 juin & Paruline à gorge orangée & Dendroica fusca & M & 1 & Nord-est \\
\hline 13,15 juin & Aucun & & & & \\
\hline \multirow[t]{2}{*}{16 juin } & Cardinal à poitrine rose & Pheucticus ludovicianus & M & 1 & Nord-est \\
\hline & Viréo aux yeux rouges & Vireo olivaceus & & 1 & Nord-est \\
\hline 19 juin & Grive des bois & Hylocichla mustelina & & 1 & Sud-est \\
\hline 23,29 juin & Aucun & & & & \\
\hline \multirow[t]{2}{*}{1 juil. } & Grive & Catharus sp. & & 1 & \\
\hline & Bruant & & & 1 & \\
\hline 3 juillet & Paruline jaune & Dendroica petechia & M & 1 & Nord-est \\
\hline 5 juillet & Aucun & & & & \\
\hline 6 juillet & Quiscale bronzé & Quiscalus quiscula & & 1 & Nord-est \\
\hline 7 juillet & Aucun & & & & \\
\hline \multirow[t]{2}{*}{10 juillet } & Pic flamboyant & Colaptes auratus & & 1 & \\
\hline & Quiscale bronzé & Quiscalus quiscala & & 1 & Nord-est \\
\hline 11 juillet & Paruline obscure & Vermivora peregrina & & 1 & Nord-est \\
\hline \multicolumn{6}{|l|}{1980} \\
\hline \multirow{5}{*}{15 septembre } & Paruline à joues grises & Vermivora ruficapilla & & 2 & Nord-est \\
\hline & Paruline à gorge noire & Dendroica virens & & 1 & Nord-est \\
\hline & Paruline à ailes dorées & Vermivora chrysoptera & M & 1 & Nord-est \\
\hline & Pic à dos noir & Picoides arcticus & $\mathrm{F}$ & 1 & Nord \\
\hline & Roitelet à couronne dorée & Regulus satrapa & $\mathrm{F}$ & 1 & Nord-est \\
\hline \multirow[t]{11}{*}{20 septembre } & Paruline obscure & Vermivora peregrina & & 2 & Nord-est \\
\hline & Paruline à collier & Parula americana & $\mathrm{F}$ & 1 & Nord-est \\
\hline & Paruline des ruisseaux & Seiurus noveboracensis & & 1 & Nord-est \\
\hline & Paruline à joues grises & Vermivora ruficapilla & & 2 & Nord-est \\
\hline & Junco ardoisé & Junco hyemalis & & 3 & Sud-est \\
\hline & Gros-bec errant & Coccothraustes vespertinus & & 1 & Nord-est \\
\hline & Bruant à gorge blanche & Zonotrichia albicollis & M & 3 & Nord-est \\
\hline & Grive solitaire & Catharus guttatus & & 1 & Nord-est \\
\hline & Grive à dos olive & Catharus ustulatus & & 1 & Nord-est \\
\hline & Mésange à tête noire & Parus atricapillus & & 2 & Nord-est \\
\hline & Roitelet à couronne dorée & Regulus satrapa & M & 1 & Nord-est \\
\hline \multirow{16}{*}{$\begin{array}{c}8,910,14,15 \text { octobre } \\
\text { (Rosa, 1980) }\end{array}$} & Junco ardoisé & Junco hyemalis & & 21 & \# \\
\hline & Gros bec errant & Coccothraustes vespertinus & & 18 & \# \\
\hline & Roitelet à couronne dorée & Regulus satrapa & & 7 & \# \\
\hline & Paruline à joues grises & Vermivora ruficapilla & & 5 & \# \\
\hline & Paruline & & & 4 & \# \\
\hline & Paruline à croupion jaune & Coccothraustes vespertinus & & 3 & \# \\
\hline & Bruant hudsonien & Spizella arborea & & 3 & \# \\
\hline & Grive à dos olive & Catharus ustulatus & & 3 & \# \\
\hline & Grive solitaire & Catharus guttatus & & 2 & \# \\
\hline & Roitelet à couronne rubis & Regulus calendulus & & 1 & \# \\
\hline & Paruline bleue & Dendroica caerulenscens & & 1 & \# \\
\hline & Paruline masquée & Geothlypis trichas & & 1 & \# \\
\hline & Paruline à calotte noire & Wilsonia pusilla & & 1 & \# \\
\hline & Cardinal à poitrine rose & Pheucticus ludovicianus & & 1 & \# \\
\hline & Mésange à tête noire & Poecile atricapillus & & 1 & \# \\
\hline & Bruant à gorge blanche & Zonotrichia albicollis & & 1 & \# \\
\hline \multicolumn{6}{|l|}{2000} \\
\hline \multirow[t]{2}{*}{13 mai } & Paruline triste* & Oporornis philadelphia & M & 2 & Nord-est \\
\hline & Paruline à flancs marron & Dendroica pensylvanica & M & 1 & Nord-est \\
\hline \multirow[t]{2}{*}{14 mai } & Paruline à flancs marron & Dendroica pensylvanica & M & 1 & Nord-est \\
\hline & Paruline (tête mangée) & & & 1 & \\
\hline \multirow{3}{*}{$\begin{array}{c}15,16,17,19,21 \text { mai } \\
22 \text { mai }\end{array}$} & Aucun & & & & \\
\hline & Paruline à croupion jaune & Coccothraustes vespertinus & M & 1 & Nord-est \\
\hline & Paruline à tête cendrée & Dendroica magnolia & M & 1 & Nord-est \\
\hline 27 mai & Aucun & & & & \\
\hline 28 mai & Paruline à flancs marron & Dendroica pensylvanica & M & 1 & Nord-est \\
\hline 30 mai & Viréo aux yeux rouges & Vireo olivaceus & & 1 & Nord-est \\
\hline $3,4,11,17,18$ juin & Aucun & & & & \\
\hline
\end{tabular}


ANNEXE: (fin)

\begin{tabular}{|c|c|c|c|c|c|}
\hline Année & Espèce & Nom scientifique & Sexe & Nombre & Endroit \\
\hline 20,22 août & Aucun & & & & \\
\hline 11 octobre & Aucun & & & & \\
\hline 12 octobre & Grive solitaire* & Catharus guttatus & & 1 & \\
\hline & Bruant & & & 1 & \\
\hline & Bruant chanteur & Melospiza melodia & & 1 & \\
\hline $13,16,22$ octobre & Aucun & & & & \\
\hline \multicolumn{6}{|l|}{2001} \\
\hline 21 mai & Aucun & & & & \\
\hline 4 septembre & Aucun & & & & \\
\hline \multicolumn{6}{|l|}{2003} \\
\hline 8 octobre & Aucun & & & & \\
\hline 8 novembre & Pic chevelu & Picoides villosus & & 1 & Nord-est \\
\hline \multicolumn{6}{|l|}{2006} \\
\hline 20,22 septembre & Aucun & & & & \\
\hline 25 septembre & Paruline à croupion jaune* & Coccothraustes vespertinus & & 1 & Nord-est \\
\hline 27 septembre & Paruline à joues grises & Vermivora ruficapilla & & 1 & Nord-est \\
\hline 28 septembre & Paruline obscure & Vermivora peregrina & & 1 & Nord-est \\
\hline 2 octobre & Aucun & & & & \\
\hline 3 octobre & Roitelet à couronne rubis & Regulus calendula & & 1 & Nord-est \\
\hline $4,5,10$ octobre & Aucun & & & & \\
\hline 11 octobre & Roitelet à couronne dorée & Regulus satrapa & M & 1 & Nord-est \\
\hline $12,13,14,15$ octobre & Aucun & & & & \\
\hline 16 octobre & Grimpereau brun & Certhia americana & & 1 & Nord-est \\
\hline $17,18,24,25$ octobre & Aucun & & & & \\
\hline \multicolumn{6}{|l|}{2007} \\
\hline 20 avril & Aucun & & & & \\
\hline 17 mai & Aucun & & & & \\
\hline \multirow[t]{2}{*}{21 mai } & Paruline & & & & \\
\hline & Paruline à croupion jaune* & Coccothraustes vespertinus & M & 1 & Nord-est \\
\hline 26,27 mai & Aucun & & & & \\
\hline 9 juin & Aucun & & & & \\
\hline 1 septembre & Aucun & & & & \\
\hline $2,5,6,8,10,13$ octobre & Aucun & & & & \\
\hline 14 octobre & Roitelet à couronne rubis & Regulus calendula & M & 1 & Sud-est \\
\hline 15,16 , octobre & Aucun & & & & \\
\hline \multirow[t]{2}{*}{17 octobre } & Junco ardoisé & Junco hyemalis & M & 2 & Nord-est \\
\hline & Bruant* & & & 1 & Nord-est \\
\hline 18 octobre & Aucun & & & & \\
\hline 19 octobre & Roitelet à couronne rubis* & Regulus calendula & $\mathrm{F}$ & 2 & Nord-est \\
\hline $23,25,27$ octobre & Aucun & & & & \\
\hline
\end{tabular}

*Assommée seulement, a repris son envol

\# Façade nord-est à $92 \%$

TOTAL: 


\section{News and Comment}

\section{Marine Turtle Newsletter (121)}

July 2008. 48 pages: ARTICLES: Trade Secrets: A Ten Year Overview of the Illegal Import of Sea Turtle Products (S. M. Rice \& M. K. Moore) - In-water Observations of Recently Released Juvenile Hawksbills (Eretmochelys imbricata) (S. G. Dunbar, Lidia Salinas, \& Larry Stevenson) - Special Theme Section: - Dhamra Port Development Debate, Orissa, India Perspectives and Lessons: Editorials by Doug Hykle, Nicholas Mrosovsky, Kartik Shanker, Janaki Lenin \& Rom Whitaker, Ashish Fernandes, Sudarshan Rodriguez \& Aarthi Sridhar, Nicolas Pilcher, Holly T. Dublin, Amlan Dutta, Jack Frazier - MEETING REPORT ANNOUNCEMENTS - IUCN-MTSG - NEWS \& LEGAL BRIEFS - RECENT PUBLICATIONS - ACKNOWLEDGEMENTS.
The Marine Turtle Newsletter is edited by Lisa M. Campbell, Nicholas School of Environment and Earth Sciences, Duke University, 135 Duke Marine Lab Road, Beaufort, North Carolina 28516 USA; and Matthew H. Godfrey, NC Sea Turtle Project. North Carolina Wildlife Resources Commission, 1507 Ann Street, Beaufort, North Carolina 28516 USA. Subscriptions and donations towards the production of the MTN can be made online at http://www.seaturtle. org $/ \mathrm{mtn} /$ or postal mail to Michael S. Coyne (Managing Editor); Marine Turtle Newsletter, A321 LSRC, Box 90328 Nicholas School of Environment and Earth Sciences, Duke University, Durham, North Carolina 27708-0328 USA; e-mail: mcoyne@seaturtle.org.

\section{Errata Canadian Field-Naturalist 121(4) omission Canadian Association of Herpetologists Association canadien des herpetologists Bulletin 15(1) Fall 2007}

CONTENTS: Instructions for Authors - Editorial Notes - MEETINGS: CARCNet 20007 in Kingston, $\mathrm{ON}$ - Feature Article: Emerging infectious diseases in amphibians: Towards a concerted effort (David Lesbarreres, V. St. Amour, B. Pauli, and Tarner) - FIELD NOTES: Multiple scale habitat selection by Blanding's turtles in Algonquin Provincial Park (Christopher Edge) - BOOK REVIEW: "Naming Names: The Amphibian Tree of Life" by Frost and 18 coauthors 2006. American Museum of Natural History Bulletin 297: 1-230 (David Green, a self-review by one of the coauthors) - Thesis Abstracts in Canadian Herpetology (2007, MSc): UNIVERSITY OF OTTAWA, supervisor Gabriel Blouin-Demers: Marie-Andrée Carrière) "Movement patterns and habitat selection of common map turtles (Graptemys geographica) at St. Lawrence Islands National Park, Ontario, Canada"; LAURENTIAN UNIVERSITY (supervisor Jacqueline D. Litzgus): Jean J. Enneson "Population viability analysis and response to habitat change in Spotted Turtles (Clemmys guttata); William F. Greaves "A cold and harsh environ- ment: demography and special ecology of a northern population of Wood Turtles (Glyptemys insculpta), Dan J. Reeves Modeling critical breeding habitat and body size in the federally endangered Spotted Turtle (Clemmys guttata); UNIVERSITY OF VICTORIA (supervisor Patrick T. Gregory): Liumila (Lita) Michelle Gomez "Habitat use and movement patterns of Northern Pacific Rattlesnake (Crotalus o. oreganus) in British Columbia"; MCGILL UNIVERSITY (supervisor David M. Green): Julie A. Lee-Yaw "The phylogenetic history of the Wood Frog (Rana sylvatica); Tricia Markle "Range limitations of stream salamanders in Quebec and Labrador", Shavonne J. Meyer "Landscape history, dispersal and the genetic structure of amphibian populations" - RECENT PUBLICATIONS in Canadian Herpetology: Citations 2006-2007 - ANNOUNCEMENTS: Remembering Ted David; ASIH Special Publications - Membership Form.

The CAH-ACH Bulletin is Edited by Jacqueline D. Litzgus, Department of Biology, Laurention University, Sudbury, Ontario P3E 2C6, jlitzgus Laurnetian.ca.

\section{Errata Canadian Field-Naturalist 122(2) omission}

Pouliot, Yvan. 2008. Les collisions d'oiseaux à l'édifice Marly, St. Foy, Quebec, de 1978 à 2000, page
155. Remerciements: between Ouelett and Lessard add Pierre Richard. 